Article

\title{
Surface Modification of Poly(L-lactic acid) Nanofiber with Oligo(D-lactic acid) Bioactive-Peptide Conjugates for Peripheral Nerve Regeneration
}

\author{
Sachiro Kakinoki ${ }^{1,2}$, Sho Uchida ${ }^{1,3}$, Tomo Ehashi ${ }^{1,2}$, Akira Murakami ${ }^{3}$ and \\ Tetsuji Yamaoka ${ }^{1,2, *}$
}

1 Department of Biomedical Engineering, National Cardiovascular Center Research Institute, 5-7-1

Fujishirodai, Suita, Osaka 565-8565, Japan; E-Mails: sachiro@ri.ncvc.go.jp (S.K.);

shou_uchida@gg.nitto.co.jp (S.U.); ehashi.org@tmd.ac.jp (T.E.)

2 JST, CREST, 5 Sanbancho, Chiyoda-ku, Tokyo 102-0075, Japan

3 Department of Polymer Science and Engineering, Kyoto Institute of Technology, Matsugasaki, Sakyo-ku, Kyoto 606-8585, Japan; E-Mail: akiram@kit.ac.jp (A.M.)

* Author to whom correspondence should be addressed; E-Mail: yamtet@ $@$ ri.ncvc.go.jp;

Tel.: +81-6-6833-5012 ext. 2637; Fax: +81-6-6835-5476.

Received: 22 February 2011 / Accepted: 27 April 2011 / Published: 27 April 2011

\begin{abstract}
In some traumatic nerve injuries, autologous nerve grafting is the first choice for bridging the gap between the severed nerve ends. However, this therapeutic strategy has some disadvantages, including permanent loss of donor function and requirement of multiple surgeries. An attractive alternative to this therapeutic technique is the use of artificial nerve conduit. Poly (L-lactic acid) (PLLA) is widely used as a substrate for artificial nerve conduit because it is readily biodegradable, but it is not inherently biologically active. In this study, we developed a PLLA nanofibrous nerve conduit, modified with a conjugate of oligo (D-lactic acid) (ODLA) and the neurite outgrowth, thereby promoting peptide AG73 (RKRLQVQLSIRT) to improve nerve regeneration. PLA/ODLA-AG73 nanofibrous conduit was fabricated by electrospinning and then transplanted at the $10 \mathrm{~mm}$ gap of rat sciatic nerve. After six months, electrophysiological evaluation revealed that it achieved better functional reinnervation than silicone tube (used as a reference) or unmodified PLLA nanofibrous conduit.
\end{abstract}

Keywords: nerve regeneration; poly (L-lactic acid); oligo (D-lactic acid)-peptide conjugates; nanofibrous nerve conduit; AG73 


\section{Introduction}

Nerve injuries - especially the most serious type, neurotmesis - typically result in significant nerve gaps and loss of motor and sensory functions. If the gap is short, the severed nerve stumps can often be reconnected surgically owing to non-tension; if it is long, an autologous nerve graft from the patient's own body is used to bridge the injury site. However, autologous nerve grafts have several disadvantages, including permanent loss of donor function, requirement of multiple surgeries, and size mismatch between the injured nerve and the graft nerves. Therefore, artificial nerve conduit is of great interest for bridging the gap between severed nerve stumps [1,2].

Various biodegradable polymers are used as substrates for nerve conduit. Poly(lactic acid) (PLA) [3-5] and its copolymers are preferred because they are non-enzymatically hydrolyzed to low toxic lactic acid and are metabolized via the tricarboxylic acid cycle in vivo [6]. In addition, PLA has attractive mechanical properties and excellent shaping and molding properties, and can be fabricated into porous, nanofibrous, and many other types of structures. However, PLA exhibits poor hydrophilicity and lacks congenital biological activity, so it is usually modified with biologically active molecules. For nerve conduit, the proteins collagen and laminin are used as modifiers, because their biologically active sequences promote nerve regeneration [7]. However, these proteins are animal-derived, and can display high antigenicity because of unnecessary biologically active sequences and enzymatically digested fragments [8].

Many biologically active peptides are reported to be useful for tissue regeneration. Focusing on nerve regeneration, the peptides RGD, IKVAV, and YIGSR, isolated from fibronectin or laminin, are preferred to improve the biological activities of nerve conduit [9]. Another advantage is that, although they are biologically active, they are prepared by chemical procedures.

There are many reports of nerve conduit being fabricated with combinations of biodegradable polymer substrates (at this point, typically chitosan) and biologically active peptides. For example, a nanofibrous nerve conduit combined with YIGSR peptide has been shown to promote nerve repair in rats [10]. However, although it is easy to introduce bioactive molecules into chitosan via amino groups, it is difficult to control their biodegradability. In contrast, PLA biocompatibility can be controlled by molecular weight regulation or by copolymerization with other hydroxyl acids such as glycolic acid. However, PLA is not easily modified with bioactive molecules because it lacks functional groups.

Many investigators have attempted to add functional groups to PLA in order to enhance its biological activity via copolymerization or chemical grafting with other polymers [11], plasma treatment [12], chemical modification [13], and physical adsorption [14].

In our previous study, we developed amphiphilic oligo(D-lactic acid)-RGD (ODLA-RGD) conjugates to impart cell adhesion properties to poly(L-lactic acid) (PLLA) films [15]. The two enantiomeric PLAs, PLLA and poly(D-lactic acid) (PDLA), are known to form a stereocomplex by strong interaction between their L-lactyl and D-lactyl units [16-18]. The bioactive RGD sequence was stably immobilized onto PLLA scaffolds via stereocomplex formation of ODLA segment and PLLA, resulting in improved $3 \mathrm{~T} 3$ cell adhesion.

PLLA nanofibrous nerve conduit was modified with a novel conjugate composed of ODLA and neurite outgrowth, thereby promoting peptide AG73 (RKRLQVQLSIRT) has been fabricated by 
electrospinning PLLA and ODLA-AG73 conjugate solutions [19]. AG73 found in the 1 chain C domain of laminin-I is known to strongly promote cell adhesion, wound healing, and neurite outgrowth [20,21].

With the goal of developing novel PLLA nanofibrous nerve conduit modified with the neurite outgrowth-promoting peptide for nerve regeneration, we analyzed the surface of PLLA/ODLA-AG73 nanofibrous conduit to determine whether or not the AG73 peptides are stably immobilized on the PLLA nanofiber. We then, implanted conduit in a $10 \mathrm{~mm}$ gap in rat sciatic nerve to bridge the nerve stumps, and performed histological and electrophysiological evaluations of the repaired nerve after six months.

\section{Experimental Section}

\subsection{Materials}

The amino acid derivatives dimethylformamide (DMF), piperidine, and trifluoroacetatic acid (TFA) were purchased from Watanabe Chemical Industries (Hiroshima, Japan). PAL-PEG resin was purchased from Applied Biosystems (Foster City, CA, USA). The condensation agents 4-(4,6-dimethoxy-1,3,5-triazin-2-yl)-4-methylmorpholinium chloride n-hydrate (DMT-MM) and N-methylmorpholine (NMM) were obtained from Kokusan Chemical Company (Tokyo, Japan) and Nacalai Tesque (Kyoto, Japan), respectively. Acetic anhydrate and L-lactic acid were purchased from Wako Pure Chemical Industries (Osaka, Japan). PLLA $(\mathrm{mw}=130,000)$ was obtained from Mitsui Chemicals (Tokyo, Japan).

\subsection{Methods}

\subsubsection{Synthesis of ODLA-AG73 Conjugates}

ODLA-AG73 conjugates were synthesized on resin from 9-fluorenylmethoxycarbonyl (Fmoc) by a solid-phase procedure (Scheme 1) [22].

Scheme 1. Synthesis of ODLA-AG73 conjugates.

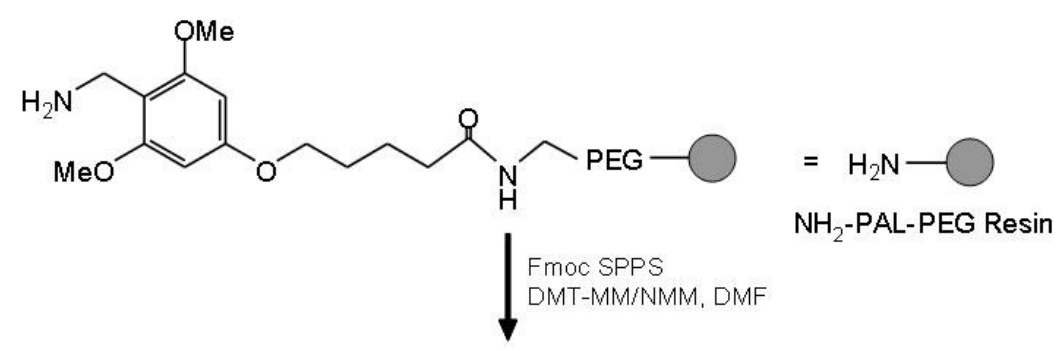

$\mathrm{NH}_{2}-\mathrm{R}(\mathrm{Pbf}) \mathrm{K}(\mathrm{Boc}) \mathrm{R}(\mathrm{Pbf}) \mathrm{LQ}(\mathrm{tBu}) \vee \mathrm{Q}(\mathrm{tBu}) \mathrm{LS}(\mathrm{tBu}) \mid \mathrm{R}(\mathrm{Pbf}) \mathrm{T}-\mathrm{NH}-$

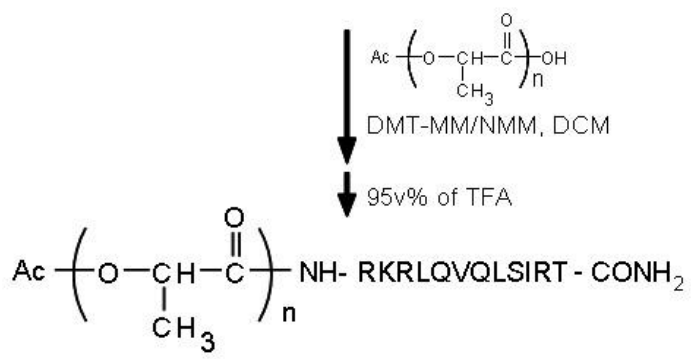


All Fmoc-protected amino acids (Fmoc-Arg(Pbf)-OH, Fmoc-Lys(Boc)-OH, Fmoc-Leu-OH, Fmoc-Gln(tBu)-OH, Fmoc-Val-OH, Fmoc-Ser(tBu)-OH, Fmoc-Ile-OH, and Fmoc-Thr-OH) were sequentially elongated on PAL-PEG resin by DMT-MM and NMM in DMF. After the coupling reaction, the absence of free amino groups was qualitatively checked by the Kaiser test. Fmoc deprotection was performed with $20 \%$ piperidine in DMF. After deprotection of the final Fmoc group, acetylated ODLA, synthesized by direct condensation polymerization, was introduced using DMT-MM and NMM in DCM. Deprotection of the side chains and ODLA-AG73 cleavage was performed with $95 \mathrm{v} \%$ TFA aqueous solution. Filtration followed by precipitation from diethyl ether yielded the ODLA-AG73 conjugate, as characterized by MALDI-TOF/MS.

\subsubsection{Fabrication of PLLA/ODLA-AG73 Nanofibrous Nerve Conduit}

PLLA/ODLA-AG73 nanofibrous nerve conduit was fabricated by electrospinning (Figure 1). For the inner layer of the conduit, PLLA/ODLA-AG73 solution was prepared at $10 \mathrm{w} \%$ in hexafluoroisopropanol (HFIP) with 3 w\% ODLA-AG73 conjugate for PLLA. For the outer layer of the conduit, $10 \mathrm{w} \%$ PLLA solution in HFIP containing $1 \mathrm{w} \%$ PEG for PLLA was prepared. Electrospinning of PLLA/ODLA-AG73 solution was performed using a plastic syringe equipped with a stainless steel needle (length $=15.0 \mathrm{~mm}$, diameter $=20 \mathrm{G}$ ) at a constant feed rate of $50 \mu \mathrm{L} / \mathrm{min}$. A rotating stainless steel tube (outer diameter $=1.0 \mathrm{~mm}$, speed $=1,500 \mathrm{rpm}$ ) was used as a target; the distance between the needle tip and the target was $100 \mathrm{~mm}$. Positive-charged PLLA/ODLA-AG73 (inner layer) and PLLA/PEG (outer layer) solutions were injected using a needle to the target at high voltage $(13 \mathrm{kV})$ for 1 and $9 \mathrm{~min}$, respectively. PLLA/ODLA-AG73 nanofibrous nerve conduit was removed from the target, cut to lengths of $12 \mathrm{~mm}$, washed with Milli-Q water and ethanol, and dried in vacuo to remove the remaining organic solvents. Surface morphology was observed by scanning electron microscopy (SEM; JCM-5700, JEOL, Tokyo, Japan).

Figure 1. Illustration of the surface structure of PLLA/ODLA-AG73 nanofiber.
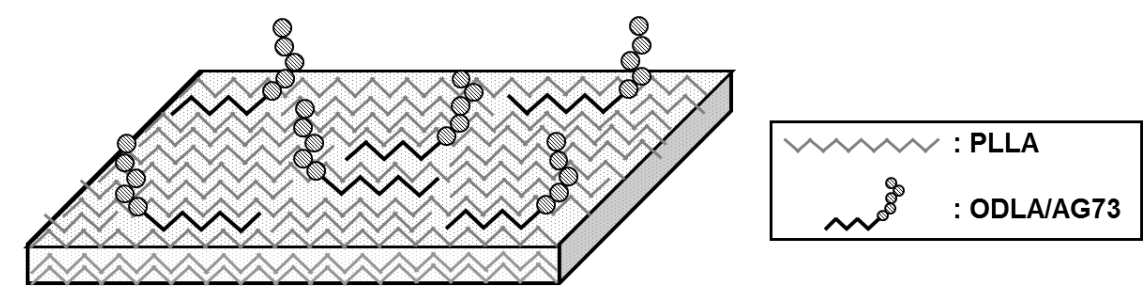

\subsubsection{Determination of Conduit Surface Composition}

The surface composition of PLLA/ODLA-AG73 nanofibrous nerve conduit was determined by X-ray photoelectron spectroscopy (XPS; ESCA-3400, Shimadzu Co., Kyoto, Japan). The X-ray source was a monochromatic $\mathrm{Mg} \mathrm{K} \alpha \mathrm{X}$-ray from a rotating anode. Survey scans were performed from 0 to $1,200 \mathrm{eV}$. Peak positions and areas were analyzed, and $\mathrm{C} 1 \mathrm{~s}, \mathrm{~N} 1 \mathrm{~s}$, and $\mathrm{O} 1 \mathrm{~s}$ ratios were calculated with the software provided by the manufacturer. 


\subsubsection{Conduit Implantation}

A total of six SD rats (seven weeks old, male) (Japan SLC, Shizuoka, Japan) were used to compare PLLA nanofibrous nerve conduit with and without ODLA-AG73 and silicone tubes $(N=2)$. Silicone tubes (length $=12 \mathrm{~mm}$, inner diameter $=1.0 \mathrm{~mm}$, outer diameter $=2.0 \mathrm{~mm})($ Sogo Laboratory Glass Works Company, Kyoto, Japan), sterilized with ethylene oxide, were used as negative control because they do not collapse and conglutinate with surrounding tissues after implantation [23].

The rats were anesthetized with Escain isoflurane (Mylan Inc., Canonsburg, PA, USA), the right sciatic nerve was exposed, and a $10 \mathrm{~mm}$ segment was removed from the distal portion of the nerve. A conduit was implanted so as to bridge the stumps of the removed nerve. The conduit was filled with physiological saline solution and sutured in place with 10-0 vicryl (Ethicon, Somerville, NJ, USA). The muscle incision and skin were closed with 3-0 silk suture (Ethicon).

\subsubsection{Histological Evaluation}

The rats were sacrificed six months after implantation and the nerve conduits, including regenerative nerve, were carefully dissected.

Specimens were fixed with $10 \%$ formalin neural buffer solution, dehydrated in ethanol series, embedded in paraffin, cut into thin cross sections $\sim 2-4 \mathrm{~mm}$ from a distal connection of $3.0 \mu \mathrm{m}$ thickness with an ultramicrotome, and stained with the following: (1) hematoxylin and eosin (HE), (2) anti-glial fibrillary acidic protein (GFAP) rabbit polyclonal antibody (Dako, Glostrup, Denmark), and (3) anti-neurofilament $200 \mathrm{kD}$ (NF200) rabbit polyclonal antibody (Sigma-Aldrich). Immunohistochemical analysis was performed by standard immunoperoxidase techniques.

After de-paraffinization, the sections were treated as follows. Sections for NF200 staining were incubated in TBS buffer solution [50 mM tris- $\mathrm{HCl}(\mathrm{pH} 7.6)$ and $0.15 \mathrm{M} \mathrm{NaCl}$ ] containing $0.65 \mathrm{mg} / \mathrm{mL}$ of proteinase $\mathrm{K}$ (Dako) for $5 \mathrm{~min}$ at room temperature and then washed with TBST buffer solution [50 mM tris- $\mathrm{HCl}$ ( $\mathrm{pH} 7.6), 0.05 \%$ Tween-20, and $0.15 \mathrm{M} \mathrm{NaCl}$; ; endogenous peroxidase activity was blocked with $3.0 \%$ hydrogen peroxide in methanol for $10 \mathrm{~min}$. Sections for GFAP and NF200 staining were incubated with an appropriate dilution of a primary antibody for $30 \mathrm{~min}$ at room temperature and overnight at $4{ }^{\circ} \mathrm{C}$, respectively. After rinsing the primary antibody using a TBST buffer, the sections were incubated for $30 \mathrm{~min}$ at room temperature with a secondary antibody (N-Histofine Simple Stain Rat MAX PO, Nichirei Biosciences, Tokyo, Japan). Then, the slides were incubated with DAB+ solution (Dako) for $30 \mathrm{~min}$ and rinsed gently with distilled water. Counterstaining was carried out with hematoxylin for $1 \mathrm{~min}$. The slides were rinsed in water until clear, dehydrated with ethanol, and washed with xylene. Finally, a coverslip was applied with Malinol (Muto Pure Chemical Company, Tokyo, Japan) and the slides were observed by light microscopy (COOLSCOPE II, Nicon, Tokyo, JAPAN).

\subsubsection{Electrophysiological Analysis}

Rats $(N=2)$ were anesthetized with Escain isoflurane (Mylan Inc.) and the implanted nerve conduits were exposed from the proximal to the distal portions of the sciatic nerve and the tibialis muscle. An electromyogram of the tibialis muscle, a measure of myogenic potential, was obtained by 
stimulating the nerve at a point proximal to the implanted nerve conduit. Stimulation was achieved by dissecting this portion of the nerve free from surrounding tissue and positioning on it a pair of platinum wire stimulating electrodes. A pair of recording electrodes was inserted into the tibialis muscle. Both stimulating and recording electrodes were connected to an electric stimulator (SEN-3401, Nihon Kohden, Tokyo, Japan) and a data acquisition system (PowerLab 8/30, ADInstruments, Colorado Springs, CO, USA). The stimulation parameters were as follows: strength $=1 \mathrm{~V}$, duration $=10 \mathrm{~s}$, pulse $=1 \mathrm{~Hz}$. The myogenic potential of the tibial muscle was amplified with PowerLab system (ADInstruments, Burlingame, CA, USA), and the average of 50 traces was recorded.

\section{Results and Discussion}

\subsection{Preparation and Characterization of PLLA/ODLA-AG73 Nanofibrous Conduit}

ODLA-AG73 conjugates were synthesized on resin from Fmoc in a solid-phase procedure. The conjugates consisted of 3- to 10-mer ODLA and AG73 sequences, with molecular weights of $\sim 2 \mathrm{kDa}$ (Figure S1).

PLLA/ODLA-AG73 nanofibrous nerve conduit was fabricated by electrospinning solutions of PLLA or PLLA/ODLA-AG73 (97:3) (Figure 2). The diameters of the PLLA and PLLA/ODLA-AG73 nanofibers were approximately 280 and $220 \mu \mathrm{m}$, respectively, and the conduits were tightly reticulate.

Figure 2. Images of different types of nerve conduit: (A) Whole image of a silicone tube (used as control) and PLLA/ODLA-AG73 nanofibrous conduit; (B,C) Scanning electron microscope (SEM) images of PLLA and PLLA/ODLA-AG73 nanofibrous nerve conduits, respectively.
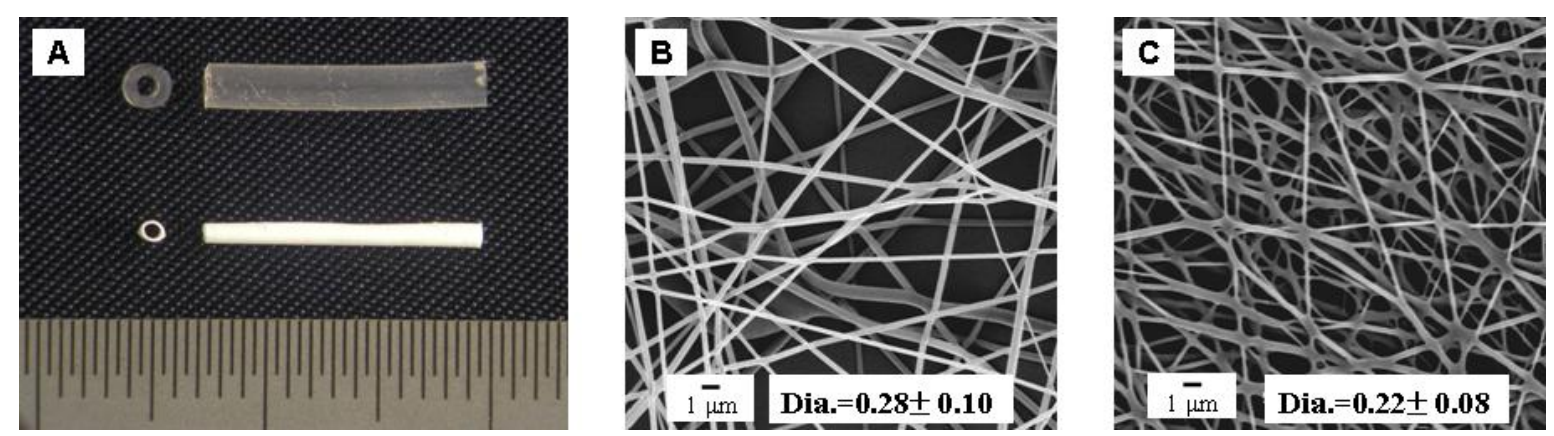

A nanofibrous conduit is thought to be the most suitable structure for suppressing connective tissue ingression and allowing permeation of liquid elements into the conduit. In our previous study, we incorporated ODLA-RGD conjugates into the PLLA crystal structure via stereocomplex formation by means of electrospinning, resulting in stable immobilization of RGD peptide on a PLLA nanofiber [15]. Similarly, we thought that we could immobilize AG73 peptides on a PLLA nanofiber using ODLA-AG73 (Figure 1). In fact, neurite outgrowth in PC12 cells (the rat pheochromocytoma cell line) is slightly better on PLLA/ODLA-AG73 nanofiber than on PLLA nanofiber in vitro (Figure S2).

To confirm that AG73 peptide was introduced onto the PLLA nanofiber using ODLA-AG73 conjugates, we analyzed the surface by XPS (Figure 3). The spectrum of the PLLA nanofibrous conduit lacks an N1s peak because the PLLA molecule does not contain nitrogen. However, after thorough 
washing, the spectrum of PLLA/ODLA-AG73 has a slight N1s peak $(\mathrm{N} 1 \mathrm{~s} / \mathrm{C} 1 \mathrm{~s}$ ratio $=0.03 \pm 0.01$ ) corresponding to amino acid, indicating that AG73 was stably immobilized on the PLLA nanofiber (Table 1). The ODLA region of the ODLA-AG73 conjugate might have incorporated or formed stereocomplex crystallites with PLLA, as reported in our previous study [15]. Because AG73 is hydrophilic, AG73 at the nanofiber surface must be exposed to liquid in order to express its biological activity in a physiological environment.

Figure 3. X-ray photoelectron spectroscopy (XPS) spectra of PLLA and PLLA/ODLA-AG73 nerve conduits. The inserted figure shows the spectra in the N1s region.

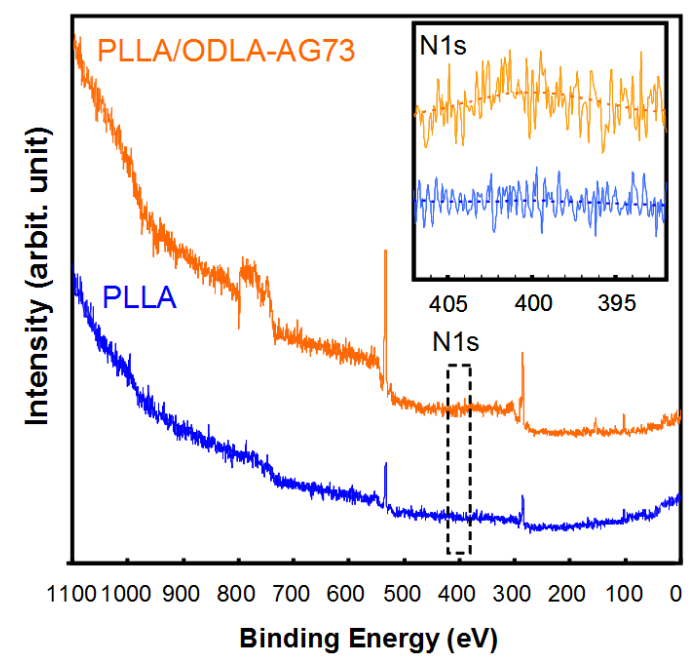

Table 1. Elemental ratios of nitrogen to carbon $(\mathrm{N} 1 \mathrm{~s} / \mathrm{C} 1 \mathrm{~s})$ and oxygen to carbon $(\mathrm{O} 1 \mathrm{~s} / \mathrm{C} 1 \mathrm{~s})$ at the surface of PLLA and PLLA/ODLA-AG73 nerve conduits, measured by XPS $(n=3)$.

\begin{tabular}{ccc}
\hline & N1s/C1s & O1s/C1s \\
\hline PLLA & N.D. & $1.23 \pm 0.08$ \\
PLLA-ODLA/AG73 & $0.03 \pm 0.01$ & $1.57 \pm 0.27$ \\
\hline
\end{tabular}

\subsection{Evaluation of Nerve Regeneration}

Silicon tubes (used as a control), PLLA fibrous conduit, and PLLA/ODLA-AG73 fibrous conduit were implanted at the $10 \mathrm{~mm}$ gap of rat peripheral nerve (Figure 4(A,C,E)). Both nerve stumps were pulled inside the tube and sutured. After six months, regenerated nerve-like tissue was visually observed inside the semitransparent silicone tube. In contrast, the PLLA and PLLA/ODLA-AG73 conduits were almost degraded and the boundaries between nerve and conduit were barely distinguishable. The silicone tube allowed the peripheral nerve to be exteriorized easily (Figure 4(B)), but the PLLA and PLLA/ODLA-AG73 conduits adhered severely to the surrounding tissue and muscle (Figure 4(D,F)). Woo et al. reported that the nanofibrous architecture of PLA scaffolds enhances the adsorption of cytoadherence proteins such as fibronectin and vitronectin, and allows cell attachment [24]. Accordingly, the PLLA and PLLA/ODLA-AG73 conduits might have promoted protein adsorption, and thus, adhesion of surrounding tissue.

We performed histological analyses of cross sections near the distal connection. Figure 5(A-C) shows the case for a healthy nerve. Figure 5(D-F) shows the case for a silicone tube (used as a 
control). GFAP-positive cells and NF200-positive axons appear at the center, resulting in overlap. The regenerating nerve is round in shape and lacks ingression of connective tissue because the tube is not biodegradable and does not allow permeation of liquid elements and cells from outside and neural-cell attachment inside the tube. Figures $5(\mathrm{G}-\mathrm{I})$ and $5(\mathrm{~J}-\mathrm{L})$ show the cases for PLLA conduit and PLLA/ODLA-AG73 conduit, respectively. In both cases, the regenerating nerve is also visible, the appearance of GFAP-positive cells and NF200-positive axons is similar, and fragments of degraded conduit are evident around the regenerating nerve. Ingression of connective tissue between the nerve stumps inhibits nerve regeneration, so it is necessary to control the degradation ratio of the conduit.

Figure 4. Intraoperative photographs of different types of nerve conduit (top row, immediately after implantation; bottom row, six months after implantation): (A,B) Silicone tube (used as a control); (C,D) PLLA conduit; (E,F) PLLA/ODLA-AG73 conduit.
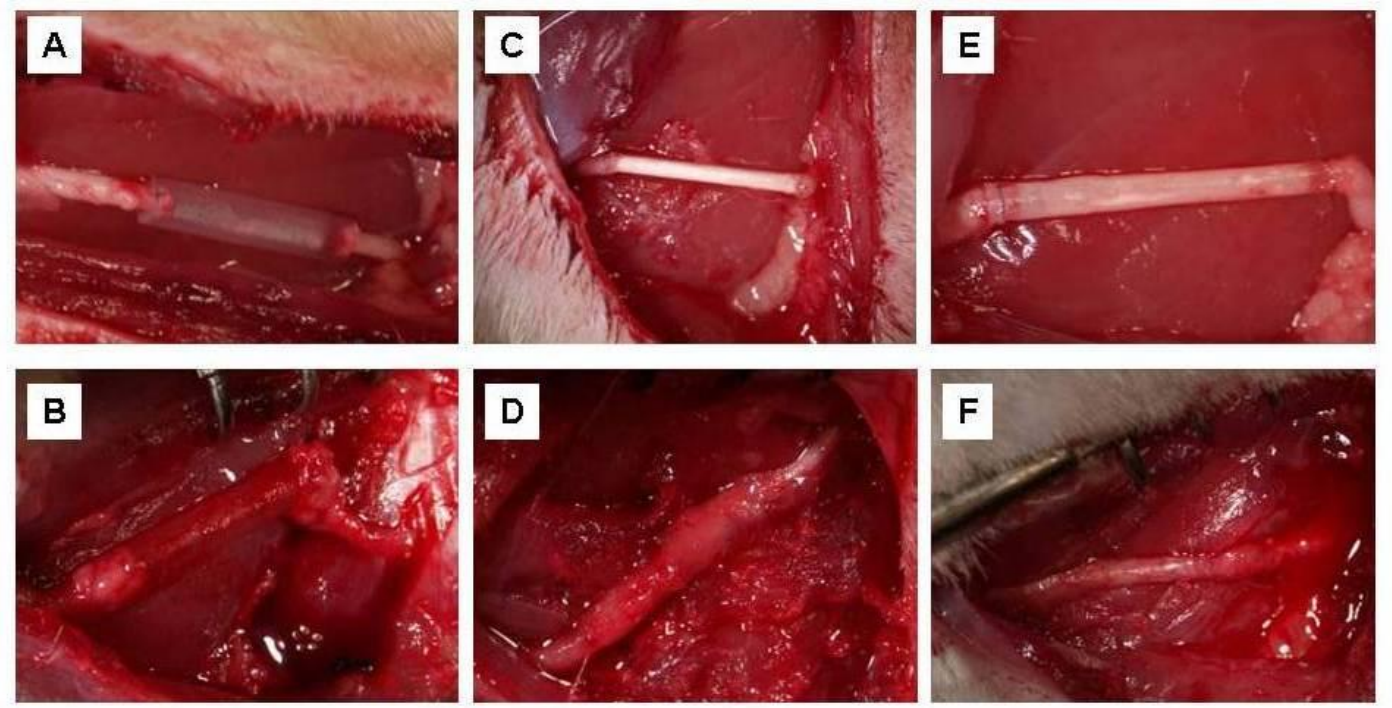

Figure 5. Immunohistochemical analysis of axial cross sections of implanted silicone tube (used as a control), PLLA conduit, and PLLA/ODLA-AG73 conduit around a distal connection six months after implantation, with different stains applied: $(\mathbf{A}, \mathbf{D}, \mathbf{G}, \mathbf{J}) \mathrm{HE}$ stain; (B,E,H,K) GFAP-positive cells (brown); (C,F,I,L) Neurofilament (brown). Note the conduit fragments (indicated by arrows) (Scale bar $=200 \mu \mathrm{m}$ ).

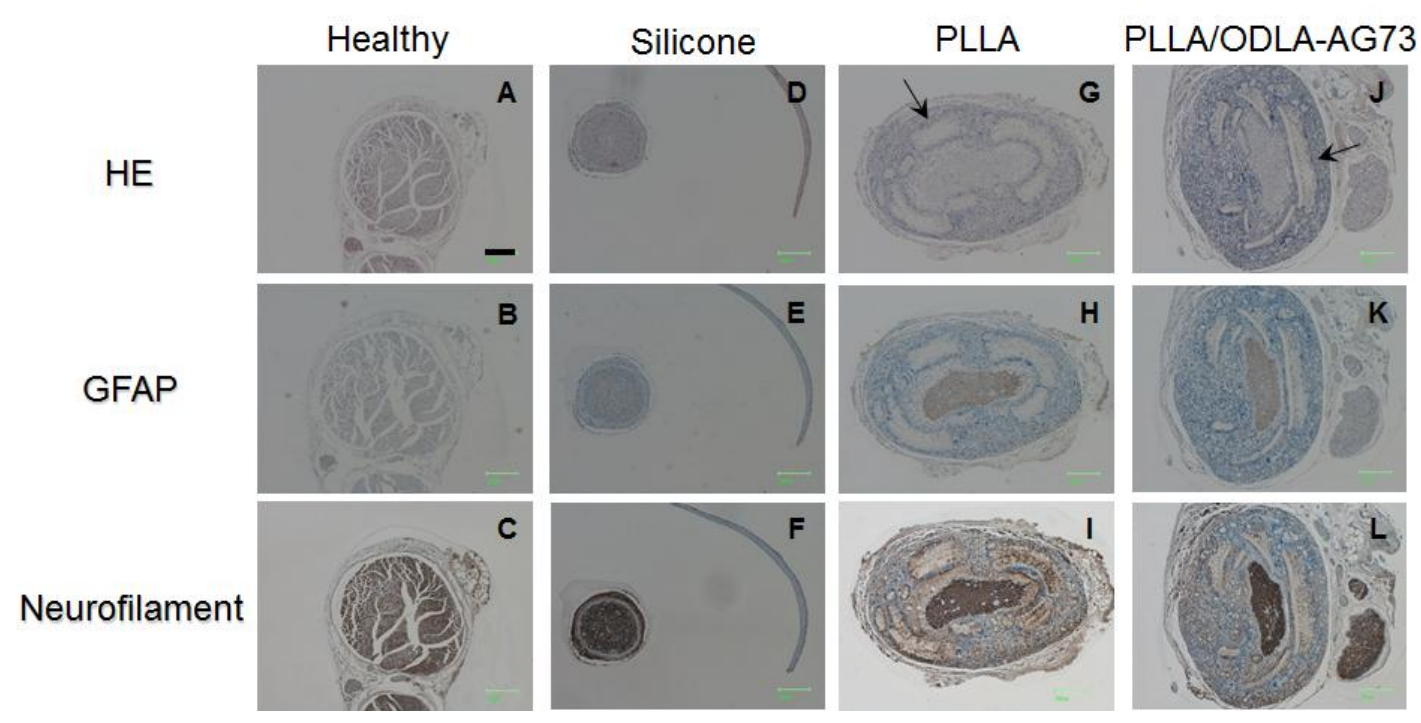


We performed electrophysiological analyses to evaluate functional reinnervation through the conduits. Figure 6(A) shows the case for a healthy nerve; the distal latency is $0.0015 \mathrm{~ms}$ and amplitude is $0.96 \mathrm{~V}$. Figure 6(B,C) shows the cases for the silicone tube and PLLA conduit, respectively. The myogenic potential of the tibialis muscle is not detectable, suggesting that the functional reinnervation was not established within the measured time. Figure 6(D) shows the case for the PLLA/ODLA-AG73 conduit. The myogenic potential pattern is similar to that for healthy nerve, indicating that the PLLA/ODLA-AG73 conduit achieves enhanced functional reinnervation. However, its distal latency $(0.0031 \mathrm{~ms})$ is longer than that of a healthy nerve because of delayed axonal remyelination [25,26]. As in Figure 5(K), more GFAP-positive non-myelinating Schwann cells [27] are observed in the PLLA/ODLA-AG73 conduit than in a healthy nerve. In addition, the myogenic potential $(6.60 \mathrm{~V})$ is much larger than that for a healthy nerve, perhaps because unwanted reinnervation between a sciatic nerve and surrounding muscle occurs when the conduit degrades and loses its barrier function. Although two rats were used for the electrophysiological analysis of the PLLA/ODLA-AG73 conduit, electromyograms of both were similar. Thus, our results suggest that the PLLA/ODLA-AG73 conduit enhances nerve regeneration and its degradation rate should be controlled to achieve functional reinnervation.

Figure 6. Electrophysiological analysis of sciatic nerves six months after implantation: (A) Healthy nerve; (B) Nerve with implanted silicone tube (used as a control); (C) Nerve with implanted PLLA conduit; (D) Nerve with implanted PLLA/ODLA-AG73 conduit.
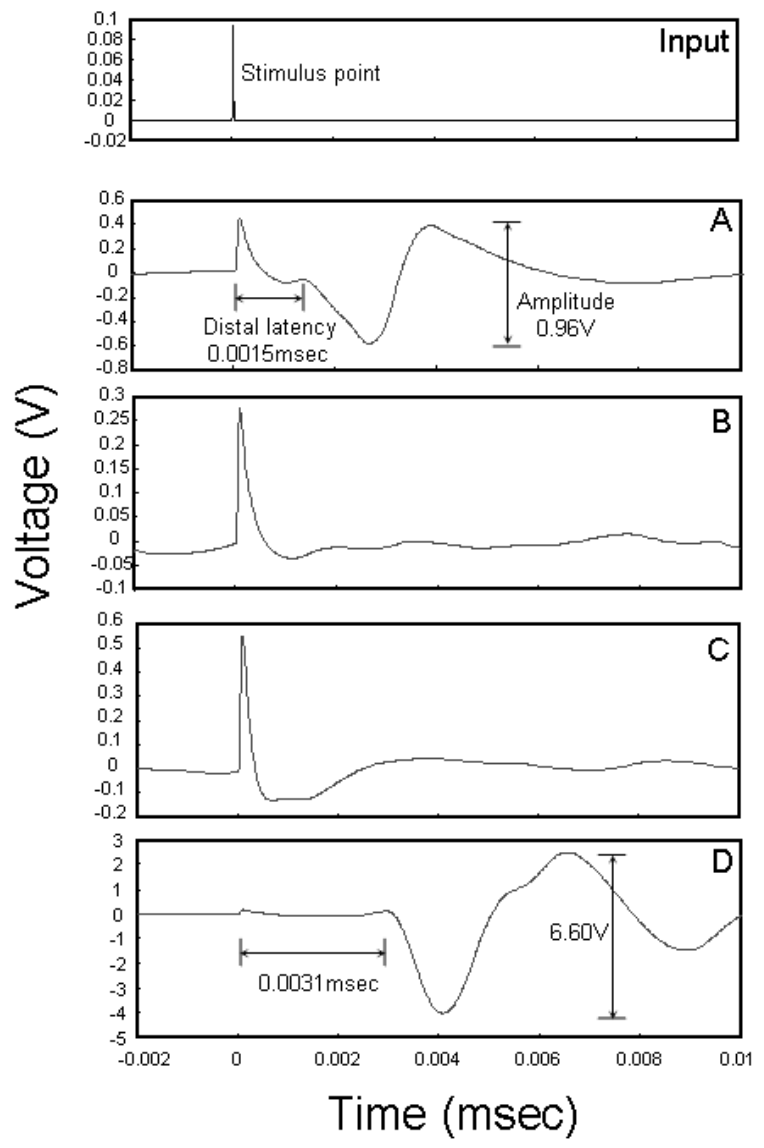

\section{Conclusions}

We fabricated PLA nanofibrous nerve conduit modified with ODLA-AG73 conjugate by electrospinning, implanted them into rat sciatic nerves, and observed the subsequent regeneration. XPS 
analysis shows that neurite outgrowth-promoting peptide AG73 was stably immobilized on the PLLA nanofiber. Electrophysiological analysis in vivo shows that the PLLA/ODLA-AG73 conduit achieved enhanced functional reinnervation. The degradation ratio of the PLLA/ODLA-AG73 conduit should be optimized to suppress unwanted reinnervation with the surrounding muscles and to develop further suitable conduit for clinical applications.

\section{Acknowledgement}

A part of this work was supported by Grant-in-Aid for Scientific Research on Innovation Areas (20106014).

\section{References}

1. Hadlock, T.; Elisseeff, J.; Langer, R.; Vacanti, J.; Cheney, M. A tissue-engineered conduit for peripheral nerve repair. Arch. Otolaryngol. Head Neck. Surg. 1998, 124, 1081-1086.

2. Meek, M.F.; Coert, J.H. Clinical use of nerve conduits in peripheral-nerve repair: Review of the literature. J. Reconstr. Microsurg. 2002, 18, 97-110.

3. Mligiliche, N.L.; Tabata, Y.; Kitada, M.; Endoh, K.; Okamoto, K.; Fujimoto, E.; Ide, C. Poly lactic acid-caprolactone copolymer tube with a denatured skeletal muscle segment inside as a guide for peripheral nerve regeneration: A morphological and electrophysiological evaluation of the regenerated nerves. Anat. Sci. Int. 2003, 78, 156-161.

4. Sundback, C.; Hadlock, T.; Cheney, M.; Vacanti, J. Manufacture of porous polymer nerve conduits next term by a novel low-pressure injection molding process. Biomaterials 2003, 24, 819-830.

5. Oh, S.H.; Kim, J.H.; Song, K.S.; Jeon, B.H.; Yoon, J.H.; Seo, T.B.; Namgung, U.; Lee, I.W.; Lee, J.H. Peripheral nerve regeneration within an asymmetrically porous PLAG/Pluronic F127 nerve guide conduit. Biomaterials 2008, 29, 1601-1609.

6. Sakai, Y.; Matsuyama, Y.; Takahashi, K.; Sato, T.; Hattori, T.; Nakashima, S.; Ishiguro, N. New artificial nerve conduits made with photocrosslinked hyaluronic acid for peripheral nerve regeneration. Bio-Med. Mater. Eng. 2007, 17, 191-197.

7. Inada, Y.; Morimoto, S.; Moroi, K.; Endo, K.; Nakamura, T. Surgical relief of causaligia with an artificial nerve guide tube: Successful surgical treatment of causalgia (Complex regional pain syndrome type II) by in situ tissue engineering with a polyglicolic acid-collagen tube. Pain 2005, 117, 251-258.

8. Andair-Kirk, T.L.; Senior, R.M. Fragments of extracellular matrix as amediators of inflammation. Int. J. Biochem. Cell Biol. 2008, 40, 1101-1110.

9. Itoh, S.; Suzuki, M.; Yamaguchi, I.; Takakuda, K.; Kobayashi, H.; Shinomiya, K.; Tanaka, J. Development of a nerve scaffold using a tendon chitosan tube. Artif. Organs 2003, 27, 1079-1088.

10. Wang, W.; Itoh, S.; Matuda, A.; Aizawa, T.; Demura, M.; Ichinose, S.; Shinomiya, K.; Tanaka, J. Enhanced nerve regeneration through a bilayered chitosan tube: The effect of introduction of glycine spacer into the CYIGSR sequence. J. Biomed. Mater. Res. 2008, 85A, 919-928.

11. Jiao, Y.P.; Cui, F.Z. Surface modification of polyester biomaterials for tissue engineering. Biomed. Mater. 2007, 2, R24-R37. 
12. Khorasani, M.T.; Mirzadeh, H.; Irani, S. Plasma surface modification of poly(L-lactic acid) and poly(lactic-co-glycolic acid) films for improvement of nerve cells adhesion. Radiat. Phys. Chem. 2008, 77, 280-287.

13. Ma, Z.; Gao, C.; Ji, J.; Shen, J. Protein immobilization on the surface of poly-L-lactic acid films for improvement of cellular interactions. Eur. Polym. J. 2002, 38, 2279-2284.

14. Kakinoki, S.; Yamaoka, T. Stable modification of poly(lactic acid) surface with neurite outgrowth-promoting peptides via hydrophobic collagen-like sequence. Acta Biomater. 2010, 6, 1925-1930.

15. Yamaoka, T.; Uchida, S.; Higami, T.; Murakami, A. Immobilization of Bioactive Molecules onto PLLA Porous Matrices for Tissue Regeneration. In Proceedings of International Chemical Congress of Pacific Basin Societies, Hawaii, HI, USA, 15-20 December 2005; Program No. 482.

16. Ikada, Y.; Jamshidi, K.; Tsuji, H.; Hyon, S.H. Stereocomplex formation between enantiomeric poly(lactides). Macromolecules 1987, 20, 904-906.

17. Fujiwara, T.; Mukose, T.; Yamaoka, T.; Yamane, H.; Sakurai, S.; Kimura, Y. Novel thermo-responsive formation of a hydrogel by stereo-complexation between PLLA-PEG-PLLA and PDLA-PEG-PDLA block copolymers. Macromol. Biosci. 2001, 1, 204-208.

18. Tsuji, H. Poly(lactide) stereocomplexes: Formation, structure, properties, degradation, and applications. Macromol. Biosci. 2005, 5, 569-597.

19. Bhardwaj, N.; Kundu, S.C. Electrospinning: A fascinating fiber fabrication technique. Biotechnol. $A d v$. 2010, 28, 325-347.

20. Weeks, B.S.; Nomizu, M.; Ramachandran, R.S.; Yamada, Y.; Kleinman, H.K. Laminin-1 and the RKRLQVQLSIRT Laminin-1 $\alpha 1$ globular domain peptide stimulate matrix metalloproteinase secretion by PC12 cells. Exp. Cell Res. 1998, 243, 375-382.

21 Mochizuki, M.; Kadoya, Y.; Wakabayashi, Y.; Kato, K.; Okazaki, I.; Yamada, M.; Sato, T.; Sakairi, N.; Nishi, N.; Nomizu, M. Laminin-1 peptide-conjugated chitosan membranes as a novel approach for cell engineering. FASEB J. 2003, 17, 875-877.

22. Weng, C.C.; Peter, D.W. Fmoc Solid Phase Peptide Synthesis; Oxford University Press: New York, NY, USA, 2000; pp. 61-62.

23. Francel, P.C.; Francel, T.J.; Mackinnon, S.E.; Hertl, C. Enhancing nerve regeneration across a silicone tube conduit by using interposed short-segment nerve grafts. J. Neurosurg. 1997, 87, 887-892.

24. Woo, K.M.; Chen, V.J.; Ma, P.X. Nano-fibrous scaffolding architecture selectively enhances protein adsorption contributing to cell attachment. J. Biomed. Mater. Res. 2003, 67A, 531-537.

25. Navarro, X.; Rodriguez, F.J.; Labrador, R.O.; Buti, M.; Ceballos, D.; Gomez, N.; Guadras, J.; Perego, G. Peripheral nerve regeneration through bioresorbable and durable nerve guides. J. Peripher. Nerv. Syst. 1996, 1, 53-64.

26. Ahmed, M.R.; Vairamuthu, S.; Shafiuzama, M.D.; Basha, S.H.; Jayakumar, R. Microwave irradiated collagen tubes as a better matrix for peripheral nerve regeneration. Brain Res. 2005, 1046, 55-67.

27. Jessen, K.R.; Morgan, L.; Stewart, H.J.S.; Mirsky, R. Tree markers of adult non-myelin-forming Schwann cells, 217c(Ran-1), A5E3 and GFAP: development and regulation by neuron-Schwann cell interactions. Development 1990, 109, 91-103. 


\section{Appendix}

Figure S1. MALDI-TOF/MS spectra of AG73, ODLA, and ODLA-AG73 conjugate. Molecular weights were determined by MALDI-TOF/MS (4800 MALDI TOF/TOF Analyzer, Applied Biosystems, Foster City, CA, USA). The molecular weight of AG73, 1,496.98 $\mathrm{Da}$, corresponds to the theoretical value. The molecular weight of ODLA ranges from about 700 to $1,750 \mathrm{Da}$, and after condensation with AG73 and ODLA, changes to about $1,750-2,150$ Da.

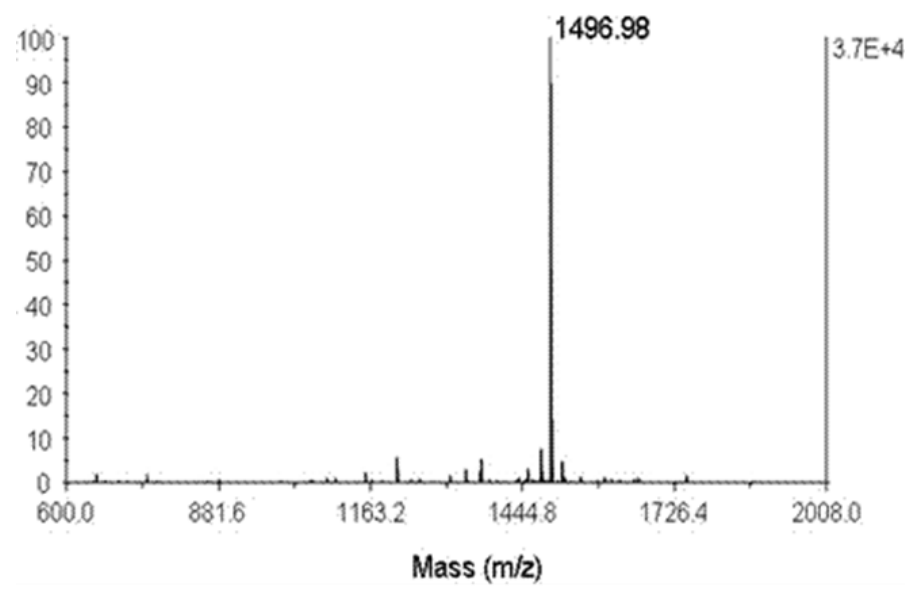

AG73

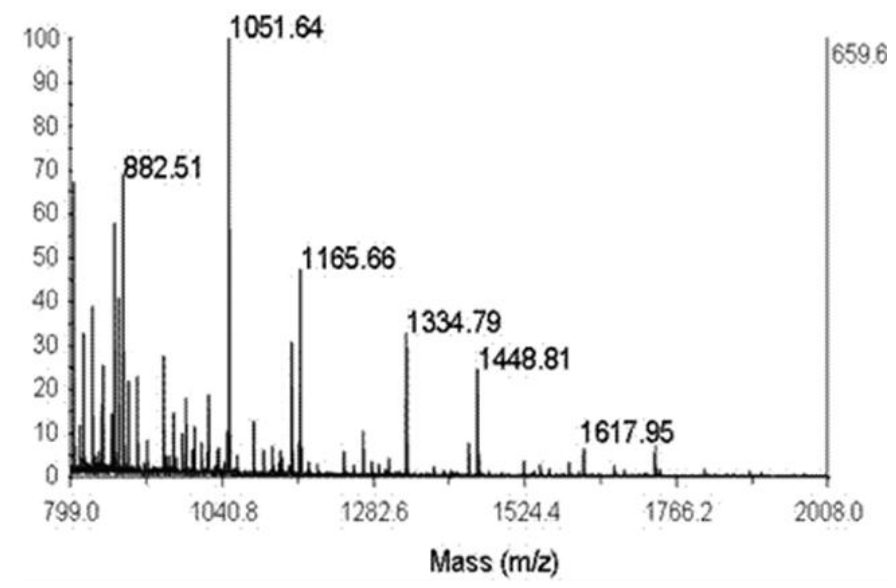

ODLA

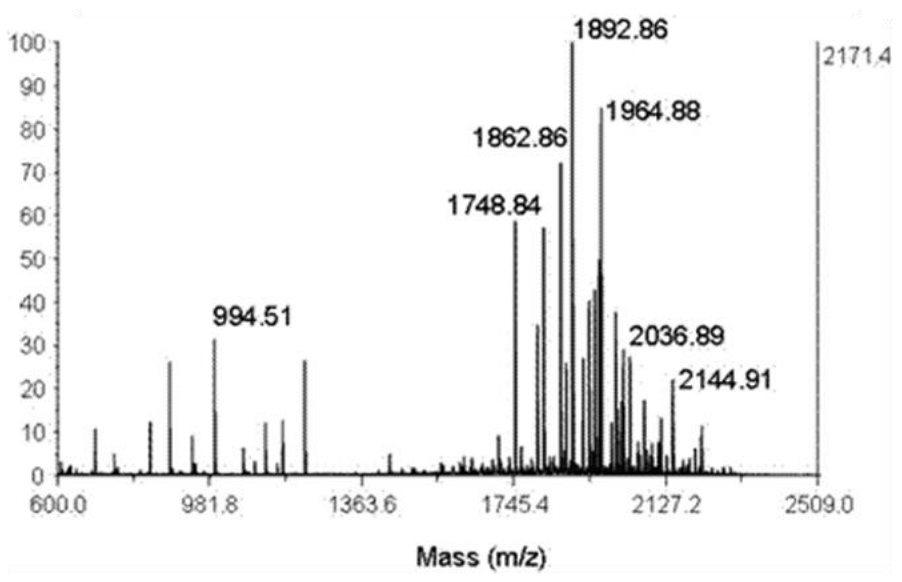


Figure S2. Adhesion and neurite outgrowth of PC12 cells on spin-coated film and on electrospun nanofibrous nonwoven PLLA or PLLA/ODLA-AG73 (scale bar $=200 \mu \mathrm{m}$ ). Spin-coated films were prepared on a glass slide with a spin coater as follows: $10 \mathrm{w} / \mathrm{v} \%$ HFIP solution of PLLA or PLLA/ODLA-AG73 (including 3 w\% ODLA-AG73 conjugate) was dropped on a slide and spread with a spin coater $(3,000 \mathrm{rpm}, 20 \mathrm{~s})$. Nanofibrous nonwoven PLLA or PLLA/ODLA-AG73 and nanofibrous conduits were fabricated on the slide by electrospinning. PC12 cells were primed with NGF for $24 \mathrm{~h}$, then, added to the samples. The samples were incubated at $37{ }^{\circ} \mathrm{C}$ for $24 \mathrm{~h}$ in Dulcecco's modification of Eagle's medium (DMEM) containing NGF. On spin-coated film, PLLA/ODLA-AG73 promoted the adhesion and neurite ourgrowth of PC12 cells. On nanofibrous nonwoven PLLA and PLLA/ODLA-AG73, the PC12 cells were slightly spread and more differentiated on PLLA/ODLA-AG73 than on PLLA.
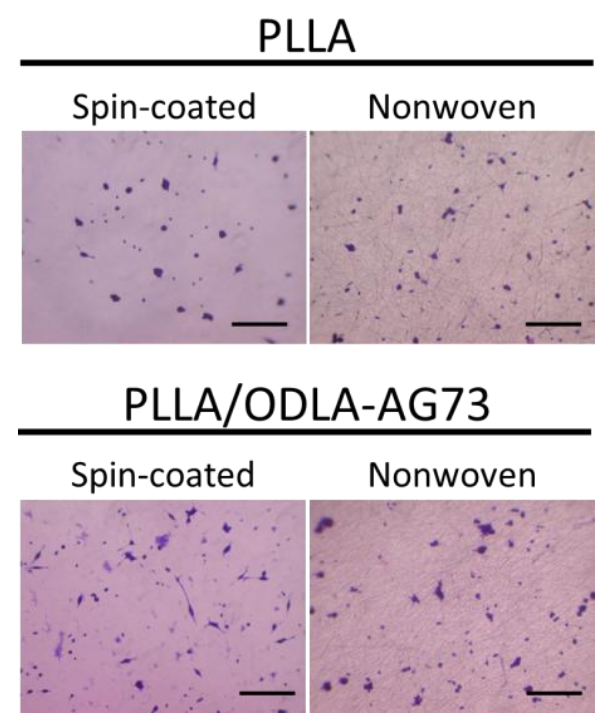

(C) 2011 by the authors; licensee MDPI, Basel, Switzerland. This article is an open access article distributed under the terms and conditions of the Creative Commons Attribution license (http://creativecommons.org/licenses/by/3.0/). 